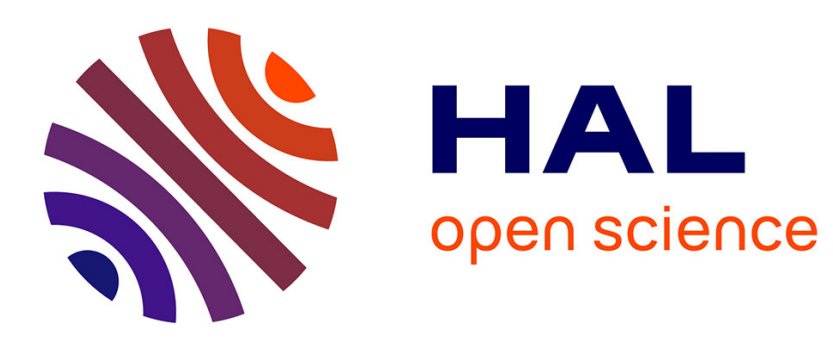

\title{
Un musée d'anthropologie oublié: le cabinet phrénologique de Dumoutier
}

Marc Renneville

\section{To cite this version:}

Marc Renneville. Un musée d'anthropologie oublié: le cabinet phrénologique de Dumoutier. Bulletins et Mémoires de la Société d'anthropologie de Paris, 1998, 10 (3), pp.477-484. 10.3406/bmsap.1998.2533 . halshs-00130223

\section{HAL Id: halshs-00130223 \\ https://shs.hal.science/halshs-00130223}

Submitted on 15 Mar 2018

HAL is a multi-disciplinary open access archive for the deposit and dissemination of scientific research documents, whether they are published or not. The documents may come from teaching and research institutions in France or abroad, or from public or private research centers.
L'archive ouverte pluridisciplinaire HAL, est destinée au dépôt et à la diffusion de documents scientifiques de niveau recherche, publiés ou non, émanant des établissements d'enseignement et de recherche français ou étrangers, des laboratoires publics ou privés. 


\section{Un musée d'anthropologie oublié : le cabinet phrénologique de} Dumoutier

Marc Renneville

Citer ce document / Cite this document :

Renneville Marc. Un musée d'anthropologie oublié : le cabinet phrénologique de Dumoutier. In: Bulletins et Mémoires de la Société d'anthropologie de Paris, Nouvelle Série. Tome 10 fascicule 3-4, 1998. pp. 477-484;

doi : 10.3406/bmsap.1998.2533

http://www.persee.fr/doc/bmsap_0037-8984_1998_num_10_3_2533

Document généré le 30/05/2016 
Bull. et Mém. de la Société d'Anthropologie de Paris, n.s., t. 10, 1998, 3-4, p. 477-484.

\title{
UN MUSÉE D'ANTHROPOLOGIE OUBLIÉ: LE CABINET PHRÉNOLOGIQUE DE DUMOUTIER
}

\author{
Marc Renneville ${ }^{1}$
}

Le texte de Pierre-Marie-Alexandre Dumoutier (1797-1871) reproduit ici est le discours qu'il prononça le jeudi 14 janvier 1836 lors de la réunion publique marquant l'inauguration du musée de la Société phrénologique de Paris(2). Ce document inédit nous rappelle l'existence dans la première moitié du $\mathrm{XIX}^{\mathrm{e}}$ siècle de ce qui fut probablement le premier Musée d'anthropologie indépendant du cabinet d'anatomie comparée du Muséum d'Histoire naturelle. Le souvenir de ce lieu d'exposition s'est bien vite effacé de la mémoire disciplinaire de l'anthropologie, car il dépendait d'une conception du fonctionnement cérébral qui fut rejetée dans la seconde moitié du XIX ${ }^{e}$ siècle. La théorie fondée par François-Joseph Gall (1758-1828) et défendue par ses disciples prétendait fonder une nouvelle anthropologie, qui résolvait définitivement le mystère des relations du physique et du moral de l'homme. Forte de ses appuis dans la philanthropie libérale de l'époque, qui a les faveurs du régime orléaniste, la phrénologie a connu sous la Monarchie censitaire une brève période de gloire, qui a commencé avec la création au début de l'année 1831 de la Société phrénologique, pour s'achever au début des années quarante. En cette même année 1836, François Broussais (1772-1838) triomphe dans un cours public de phrénologie. Conciliant la cranioscopie qui consiste à palper la boîte crânienne pour en reconnaître les saillies et les méplats et enquête "psychologique » permettant de confirmer le degré d'expression des penchants et talents détectés par la première méthode, les phrénologistes de l'époque ne perdaient pas une occasion de défendre leur théorie sur des études de cas. Les séances annuelles de la Société phrénologique proposaient alors d'étranges catafalques au public et aux curieux. Des bustes représentant criminels, fous et génies étaient

1. Département d'Histoire, Université Paris VIII, 2, rue de la Liberte, 93526 Saint-Denis Cedex. Courriel : renneville@paris7.jussieu.fr

2. Le texte manuscrit est composé de 9 feuillets. L'original est conservé aux Archives nationales dans la série AJ 15 (Muséum d' Histoire naturelle), carton $n^{\circ} 562$. Mentionné pour la première fois in Erwin Ackerknecht, «P.M.A. Dumoutier et la collection phrénologique du Musée de l'Homme », Bull. et Mém. de la Société d'Anthropologie de Paris, $10^{\circ}$ série, 7, 1956, pp. 289-308, le manuscrit de ce discours était resté jusqu'ici inédit. Pour une biographie de Dumoutier, voir cet article, ainsi que Marc Renneville, «Un terrain phrénologique dans le Grand Océan (autour du voyage de Dumoutier à bord de L'Astrolabe en 1837-40) » In: C. Blanckaert (dir.), Le terrain des sciences humaines (Instructions et enquêtes. XVIIF-XX' siècle), L'Harmattan, Paris, 1996, pp. 89-138. 
fréquemment mis en démonstration pour illustrer les 27 penchants et instincts décrits par Gall : Napoléon I Ir et l'impératrice Joséphine, Napoléon III, Liszt, le régicide Fieschi, le philosophe Auguste Comte et Vidocq subirent ainsi, parmi bien d'autres, un examen minutieux. Pendant près d'un demi-siècle, les «arpenteurs du crâne » (Esquiros) ont sillonné la France pour sonder les vices et les vertus de leur compatriotes. Leurs études de personnalités célèbres firent souvent l'objet de polémiques. Quelques-unes défrayèrent la chronique de la presse de l'époque. La séance d'inauguration du Musée fut l'occasion d'ailleurs d'une analyse phrénologique du crâne du poète assassin Lacenaire, exécuté moins d'une semaine auparavant ${ }^{(3)}$.

À la fin de l'année 1837, soit un an après sa création, le musée de la Société phrénologique de Paris avait augmenté son nombre de moulages de 200 exemplaires, le portant ainsi à 600 bustes «moulés sur nature », selon l'expression de l'époque, 300 crânes, 200 cerveaux moulés, des têtes de momie, des crânes de races et des têtes d'animaux appartenant aux quatre classes de vertébrés ${ }^{(4)}$. Ses collections ethniques furent encore notablement enrichies par la seconde circumnavigation de Jules Dumont d'Urville (18371840) auquel Dumoutier participa officiellement en tant que phrénologiste. Le musée phrénologique de Paris fut ouvert au public de 1836 à 1853 au plus tard. À cette date, le déclin irrémédiable de la théorie de Gall dans les milieux scientifiques et des difficultés financières obligèrent Dumoutier à fermer le local du 37 rue de Seine. Les collections furent entreposées à l'école de médecine, puis au Muséum d'Histoire naturelle qui les conserve aujourd'hui au Musée de l'Homme dans le Laboratoire d'anthropologie biologique. Durant la période d'ouverture du musée de Dumoutier, deux autres lieux parisiens possédaient des collections phrénologiques. Il s'agissait d'abord du cabinet d'anatomie comparée du Muséum d'Histoire naturelle, qui exposait la collection originale de François-Joseph Gall. Ce cabinet comprenait douze salles en 1836. Les pièces attractives ne manquaient pas : on pouvait observer dans la seconde et dernière salle du rez-de-chaussée le squelette de Soliman el Kaleby, assassin du général Kléber, celui du nain «Bébé» qui était au service du roi Stanislas de Pologne, celui de la Vénus Hottentote, une collection de foetus, le modèle en cire de plusieurs cas de déformations osseuses. Mais c'est en montant au premier étage que l'on pouvait admirer, après la dixième salle consacrée aux monstres et aux foetus, la collection phrénologique de Gall. L'agencement des collections, supervisé par Blainville, suivait la classification phrénologique des organes. À en croire les commentateurs de l'époque, cette salle était l'une des plus visitées par le public ${ }^{(5)}$. Le second lieu d'exposition parisien était le cabinet d'anatomie comparée de la Faculté de médecine. Composé de cinq galeries, la richesse de ses collections en faisait «l'un des

3. C'est Dumoutier qui avait fait le moulage de Lacenaire, mais c'est son collègue Hippolyte Bonnelier qui en proposa une analyse qui fut publice (Autopsie physiologique de Lacenaire, Paris, Crapelet, 1836, 65 p).

4. La Phrénologie, 1837, n²2, p. 4.

5. Céran Lemonnier et Louis Rousseau, Promenades au Jardin des Plantes, Paris, J.-B. Baillière, 1837,519 p, 1 plan et 4 figs. Les auteurs consacrent 36 pages à la description de cette salle phrénologique (pp. 100-136). 
plus beaux d'Europe». Dumoutier y avait travaille sous la Restauration comme aideanatomiste de Béclard et il avait commencé une collection de moulages de têtes de guillotinés qui était exposée dans la galerie des cires anatomiques. Les crânes de ces mêmes guillotinés se trouvaient dans la galerie consacrée aux préparations d'anatomie générale et pathologiques, aux monstruosités et aux momies ${ }^{(6)}$. S'il s'avère impossible dans l'état actuel de nos connaissances de recomposer avec précision l'agencement interne du musée de la Société phrénologique, il est probable qu'il devait suivre la division tripartite de son catalogue manuscrit : personnages célèbres, criminels, aliénés-idiots ${ }^{(7)}$. Il est certain en tout cas que les phrénologistes y voyaient un lieu d'enseignement et de démonstration populaire. Sous la Restauration déjà, Gall, Spurzheim, Fossati et Vimont avaient donné des cours publics de phrénologie. Quelques cours furent probablement dispensés également dans les grandes villes de province. Le docteur Henri-Joseph Scoutetten (1799-1871) donna en 1834 un cours public de phrénologie à Metz et le docteur A. Penot donna en 1838 à Nancy une série de conférences sur la phrénologie ${ }^{(8)}$.

Cet élan vers le public et cette volonté pédagogique - caractéristique du projet phrénologique - sont bien présents dans le discours de Dumoutier, qui donna à plusieurs reprises. Le plan de celui qu'il dispensa en 1833 en trente leçons s'organisait de la façon suivante :

" $1^{\circ}$ - description rapide de la composition anatomique de l'appareil cérébro-spinal, et du grand sympathique de l'homme et des principaux vertébrés.

$2^{\circ}$ - exposé succinct des fonctions générales de ces deux systèmes nerveux.

$3^{\circ}$ - examen minutieux de l'influence des appareils cérébraux externes sur les manifestations affectives et intellectuelles de l'homme et des animaux vertébrés qui s'en rapprochent le plus. Cet examen comprendra l'organographie phrénologique.

$4^{\circ}$ - analyse des diverses classifications les plus remarquables adoptées par les philosophes pour expliquer les actes si variés de l'homme et des animaux.

$5^{\circ}$ - application des principes de la philosophie phrénologique à l'étude de l'homme adulte considéré $1^{\circ}$ dans l'état de santé, et dans l'état pathologique, ou d'aliénation mentale, et $2^{\circ}$ dans son enfance comme sujet éducable »(9).

Une petite affiche imprimée annonça également les «cours théoriques et pratiques de phrénologie » dispensés par Dumoutier en 1836. Il était prévu 25 séances de 2 heures, données les lundis, mercredis et vendredis à partir du mercredi 25 mars, de 7 à 9 heures du soir. Dumoutier prévoyait également de faire une «démonstration de la texture du

6. G. Sarrut et Saint-Edme (Ed.), Paris pittoresque, rédigé par une société d'hommes de lettres, Paris, Au bureau de la publication, 1842, vol. II, pp. 291-292.

7. Ce catalogue est conservé au Musée de l'Homme.

8. A. Penot, Phrénologie des gens du monde (leçons publiques données à Mulhouse), Mulhouse, P. Baret, 1838.

9. Archives nationales, série $F \mathbf{1 7}$, carton $n^{\circ} 3038$. 
cerveau de l'homme », tous les jeudis de 7 à 9 heures du soir ${ }^{(10)}$. Les leçons de Dumoutier ne furent jamais publiées, à la différence de celles de Broussais père (i1).

Trois ans après la création du musée de la Société phrénologique de Paris, un certain Barthel inaugura au centre de Bruxelles un second musée entièrement consacré à la doctrine de Gall. S'annonçant professeur de «philosophie expérimentale» (ce qui était pour lui synonyme de phrénologie) et membre correspondant des sociétés «anthropologiques » de Paris et Londres, ce fouriériste considérait comme ses collègues français que l'Anthropologie était «la science de l'homme physique, moral et intellectuel au point de vue phrénologique " ${ }^{(12)}$. Les informations sur ce musée étant aussi lacunaires que pour son homologue français, il faut s'en remettre à Barthel lui-même pour sa description. "Notre musée renferme tout ce qu'il y a de plus curieux et de plus intéressant en Phrénologie. On y trouve classés les bustes ou têtes, crânes et cerveaux moulés, de près de 300 individus, morts ou vivants, qui se sont fait remarquer par de bonnes ou mauvaises qualités physiques ou organiques (appréciation faite de l'éducation etc.) sont des plus frappants [...] Difformités monstrueuses, idiots et crétins, fous ou maniaques, voleurs et assassins, suicidés même, viennent s'y montrer victimes d'une organisation vicieuse ou incomplète, que les circonstances malheureuses où ils se sont trouvés n'ont pu faire disparaître. Musiciens, Peintres et Sculpteurs, Architectes et Mathématiciens célèbres, y ont leur place à côté d'autres illustrations, tels que Poètes, Littérateurs, Philosophes et autres personnes de distinction dont la vie et les travaux sont généralement connus; quelques têtes de femmes et d'enfants, prodiges de caractère, en moralité ou en savoir, viennent, avec les crânes moulés de quelques races humaines, clore une collection Phrénologique, qu'en simple curieux comme en homme d'étude, l'on peut venir voir tous les jours »(13).

Barthel pensait que l'étude de la phrénologie et la généralisation de son enseignement devait «indubitablement amener le phénomène cérébro-moral de la Fraternité, le phénomène divin de l'Association Universelle »(14). On reconnaît ici le langage caractéristique des socialistes utopiques. Mais Barthel ne se contentait pas d'espérer et, comme Dumoutier, il était un fervent prosélyte de la «Nouvelle Anthropologie » puisqu'il donna des cours publics dans son Musée, des cours particuliers et, tous les dimanches, de 9 à 11 h du matin, des consultations phrénologiques gratuites, en français et en flamand, pour la «classe ouvrière » et «tous les individus non fortunés qui se présenteront à notre

10. Archives nationales, série AJ 15, carton $n^{\circ} 562$.

11. François-Joseph-Victor Broussais, Cours de phrénologie, Paris, J.-B. Baillière, 1836, 850 p. L'ouvrage fut réédité en 1839 sous le titre Leçons de phrénologie, Bruxelles, Société encyclographique des sciences médicales, $1839,519 \mathrm{p}$.

12. A. Barthel, Musée phrénologique de Bruxelles. Manifeste philosophique, Bruxelles, Chez tous les libraires (pp. 7-8). A l'époque, la France ne compte pas de Société anthropologique, mais une société ethnologique, à laquelle Dumoutier participera d'ailleurs, dès son retour de voyage.

13. Ibid., p. 12.

14. Ibid., p. 38 . 
établissement». Barthel prévoyait même de délivrer à chacun de ses visiteurs ouvriers « un billet, qui portera les qualités et les défauts organiques que l'examen de leur personne nous aura fait connaître, ainsi que les conseils moraux que nous aurons cru propres à corriger leur caractère ». Il se proposait également de recommander les plus méritants organiquement parlant - aux dispensateurs de bourses et au gouvernement.

Que devint le musée phrénologique de Bruxelles et la Société phrénologique que Barthel souhaitait créer en Belgique? Y eut-il en France d'autres musées phrénologiques que celui de Dumoutier? Quelle fut leur relation ${ }^{(15)}$ Le caractère privé de ces initiatives rend difficile la recherche d'éventuelles archives. Il serait intéressant pourtant de recenser les collections de bustes présentes en province. Un tel travail a été fait récemment pour Rouen, mais il faudrait l'étendre aux villes qui, comme Lyon ou Saint-Brieuc, ont connu une importante activité phrénologique. Des collections de bustes phrénologiques avaient été constituées sous la Monarchie de Juillet dans les villes de bagnes (Toulon, Rochefort, Brest) ${ }^{(16)}$. Que sont devenues ces collections ? Ont-elles été récupérées par les écoles de médecine les plus proches?

Ces collections de bustes et de crânes ont manifestement été l'objet de dédain après le déclin de la phrénologie. On pourrait s'étonner d'ailleurs qu'elles n'aient pas permis une réhabilitation partielle de la théorie de Gall dans cette seconde moitié du XIX $\mathrm{X}^{\mathrm{e}}$ siècle qui marque l'âge d'or de l'anthropologie physique française. Rares seront en effet les anthropologues qui y font allusion et si quelques criminologistes ou neurologistes la défendent, ce n'est que pour souligner l'intuition des localisations cérébrales, non la valeur du matériel accumulé. Ce hiatus peut s'expliquer. En se positionnant résolument dans le registre épistémologique de l'induction, les phrénologistes combattaient le modèle alternatif de l'auto-analyse prôné par la psychologie de Victor Cousin. Ils défendaient des faits positifs contre les déductions de leurs adversaires et voyaient dans les crânes et bustes accumulés les preuves matérielles de leurs assertions. Or l'anthropologie physique de la seconde moitié du $\mathrm{XIX}^{\mathrm{e}}$ siècle s'inscrivait dans le même système de vérité, affirmant l'objectivité des faits observés. Cette continuité du registre argumentaire ne pouvait qu'ajouter au trouble des anthropologues à venir. Rendue à la fois obsolète par l'évolution du contexte intellectuel et dangereuse par le succès même qu'elle avait rencontrée, la phrénologie fit l'objet d'un dénigrement durable. Illustrant son incapacité à en rendre compte, Paul Topinard ne voudra plus y voir en 1891 que «l'un des produits les plus étranges de l'imagination humaine», «une folie épidémique comme celle des tables toumantes " ${ }^{(17)}$. De tels jugements sont parfois reconduits euphémiquement sur l'ensemble de cette anthropologie physique du $\mathrm{XIX}^{\mathrm{e}}$ siècle

15. Des commandes de bustes provenant de New-York sont attestées par exemple pour le musée de Dumoutier (correspondance personnelle, fonds privé Colette Briot).

16. Sur les 70 bustes du Musée Flaubert et d'Histoire de la médecine de Rouen, voir Jean-Claude Vimont, 1994. Phrénologie rouennaise : les collections retrouvés, Bulletin de la Société libre d'émulation de la Seine-Maritime, pp. 39-58.

17. Paul Topinard, 1891. L'homme dans la nature, Paris, Jean-Michel Place, 1990 (préface de Nélia Dias), p. 138. 
qui encombre la mémoire de notre contemporaine «ethnologie». Il est rassurant en effet de penser que l'erreur est toujours le produit d'une déraison. Mais se rend-on compte qu'en alimentant cette historiographie, on reconduit sur le passé de l'anthropologie une attitude de dénégation qu'elle a longtemps appliquée à ses propres sujets d'observation? Les phrénologistes avaient tort, certes, mais ils forgèrent un espace de véridicité que l'historien peut rendre intelligible sans chercher à le justifier.

\section{DISCOURS LU PAR ALEXANDRE DUMOUTIER LORS DE LA SEANCE INAUGURALE DU MUSÉE DE LA SOCIÉTÉ PHRÉNOLOGIQUE DE PARIS, LE 14 JANVIER 1836}

«La phrénologie est cultivée en France, et particulièrement à Paris par un grand nombre d'hommes distingués dans la magistrature, dans les sciences, dans les arts, dans l'industrie; et la plupart de ces hommes se sont réunis pour constituer la Société phrénologique.

Cette société a adopté des époques régulières pour des réunions, elle a fait connaître les principaux résultats de ses travaux dans un journal qui forme déjà un recueil, un recueil considérable de faits curieux et d'observation utiles, enfin elle possède une collection de plus de 400 pièces et un bon nombre d'ouvrages (18). Cependant elle ne remplit encore qu'imparfaitement la mission dont elle s'est chargée : celle de poursuivre et de propager la Phrénologie.

Pour tout ceux qui l'ont étudiée et qui doivent la mettre en pratique, la Phrénologie est une science constituée qui a ses lois, ses préceptes, qui peut être réduite à un certain nombre d'axiomes et exprimée par des formules. Comme toutes les sciences exactes, elle est susceptible de la plus grande précision et peut satisfaire aux exigences du calcul et des mathématiques ${ }^{(19)}$.

Pour le philosophe et le moraliste qui a bien compris et médité les principes de la phrénologie, qui a prévu les immenses services qu'elle peut rendre par ses nombreuses applications; elle lui fournit les éléments du plus beau code de morale, et le plus beau système de philanthropie, car elle lui révèle toute la science de l'homme et lui permet de fouiller dans les replis les plus secrets de son coeur.

18. La première formule de la revue s'intitule «Journal de la Société phrénologique de Paris». A la date du discours, elle comprend trois tomes in octavo composés de dix livraisons échelonnées sur quatre années, de 1832 à 1835 dont une année d'interruption complète (1834). Le journal changera ensuite de format en 1836-37 pour paraître in quarto.

19. Une question controversée, les exigences du «calcul et des mathématiques » ayant très vraisemblablement été imposées par Francisque Lélut, qui est un adversaire de la phrénologie. 
Convaincu que je suis de l'exactitude et de l'importance de la phrénologie, me sentant capable d'opposer à ses détracteurs une masse considérable de faits, et fortifié par une expérience qui compte plus de 14 années de travaux pénibles et de sacrifices sans autres secours que la bienveillance de quelques savants que je révère et qui m'ont facilité dans la recherche des matériaux nécessaires à la démonstration de la phrénologie, j'ai péniblement amassé la riche collection étalée à vos regards.

- Maintenant et toute incomplète qu'elle est, elle peut déjà rendre de grands services. Puisque le besoin d'étudier la phrénologie se fait de plus en plus sentir chaque jour, je m'empresse de témoigner publiquement de ma profonde gratitude pour ceux qui m'ont assisté en consacrant à l'utilité publique la collection qu'ils m'ont aidé à former, et en m'adjoignant à la Société phrénologique pour vulgariser les découvertes de ses illustres prédécesseurs.

Ce n'est point assez d'une publication trimestrielle pour faire connaître les faits qui se présentent en foule; ce n'est point assez de réunions mensuelles pour donner un libre cours à l'examen et à la discussion des questions qui intéressent la science; ce n'est point assez d'une solennité annuelle pour rappeler au public l'existence de la Société phrénologique et célébrer la gloire de Gall ${ }^{(20)}$. Il y a encore d'autres devoirs à remplir et je m'y dévoue.

Voici ma moisson, voici ma fortune, je vous les offre, venez en profiter. Venez, vous qui voulez connaître, venez vous qui doutez encore, venez.

Tous les jours, les portes seront ouvertes aux travailleurs, plusieurs fois par semaine et à des heures déterminées, elles le seront aux curieux.

Par une exhibition permanente à ceux qui connaissent, j'offre des ressources qu'ils ne possèdent pas et la possibilité de faire des comparaisons sur des stéréotypes fournis par la nature. Par un enseignement permanent, je satisferai autant qu'il sera en moi de le faire à l'urgence du moment et à cet effet, pour ceux qui n'ont encore que des notions incomplètes de la phrénologie, ou qui désirent l'apprendre, quatre cours théoriques auront lieu ici et se succéderont immédiatement pendant l'année scolaire, et pour chaque leçon, toutes les démonstrations seront faites sur les objets de la collection.

Pour ceux qui ont étudié les éléments de la phrénologie mais qui ne peuvent en faire des applications exactes parce qu'ils n'ont pu être dirigé jusqu'à présent dans leurs études, et qu'ils n'ont pu trouver dans les livres de Gall et de Spurzheim les règles de la pratique et les moyens d'obtenir une évaluation exacte des diverses organisations; pour ceux-là je ferai ici des cours pratiques dans lesquels je dirigerai comme je l'ai fait précédemment chacune des personnes qui y assisteront et leur apprendrai à recueillir complètement une observation phrénologique.

Pour ceux qui cherchent à s'éclairer en offrant à leurs semblables les lumières de leur savoir et de leur raison; pour tout ceux qu'un noble enthousiasme et un sain respect pour 
la vérité invite à s'élancer dans les voies mystérieuses où s'interprète la nature morale et intellectuelle, pour tout les hommes de bien enfin, moralistes, philanthropes, artistes, gens de lettres, savants qui recherchez la vérité, pour vous des conférences seront ouvertes dans cet asile de la science. Dans ce muséum de la phrénologie pour rendre hommage à la mémoire [ " mémoire » barré et un mot illisible] de mon illustre et vénéré maître, dans ce lieu j'institue une solennité annuelle et commémorative du jour où la mort vint arracher Spurzheim des bras de ses amis et le ravir à la science qui ne s'en séparera jamais »(21).

21. Johann Gaspar Spurzheim (1776-1832) fut le principal collaborateur de François-Joseph Gall. Une brouille entre les deux hommes causa le départ de Spurzheim pour l'Ángleterre (1813), où il contribua fortement à diffuser la phrénologie. À la différence de Gall, qui s'était définitivement fixé à Paris depuis 1808, Spurzheim fut un phrénologiste itinérant. Il fonda en 1832 une éphémère Société anthropologique à Paris, et décéda la même année à Boston. L'un des paradoxes des phrénologistes français de la seconde génération est de considérer Gall comme l'unique père fondateur de leur doctrine alors qu'ils utilisent le plus souvent la carte cérébrale arrêtée par Spurzheim, qui contient 8 facultés de plus. 